\title{
Synthesis and characterization of four new unsymmetrical potentially pentadentate Schiff base ligands and related Zn(II) and Cd(II) complexes
}

\author{
Ahmad Ali Dehghani-Firouzabadi * and Fahimeh Motevaseliyan \\ Department of Chemistry, Faculty of Science, Yazd University, 89195-741 Yazd, Iran \\ ${ }^{*}$ Corresponding author at: Department of Chemistry, Faculty of Science, Yazd University, 89195-741 Yazd, Iran. \\ Tel.: +98.351.8122664. Fax: +98.351.8210644. E-mail address: aadehghani@yazd.ac.ir (A.A. Dehghani-Firouzabadi).
}

\section{ARTICLE INFORMATION}

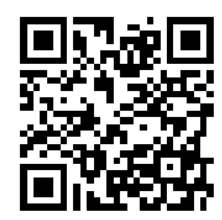

DOI: $10.5155 /$ eurjchem.5.4.635-638.1131

Received: 06 August 2014

Received in revised form: 23 August 2014

Accepted: 23 August 2014

Online: 31 December 2014

\section{KEYWORDS}

Diamine

Thioether

N202S ligands

Pentadentate ligand

Unsymmetric ligand

Schiff base complexes

\section{Introduction}

Transition metal complexes with nitrogen, oxygen and sulfur donors ligands have been prepared since the beginning of the development of coordination chemistry and there is continuing interest in these complexes [1-3]. It has attracted more attention in metal complexes of unsymmetrical Schiff bases ligands with nitrogen, oxygen and sulfur atoms in recent years due the fact that the ligands around central metal ions in natural systems are unsymmetrical $[4,5]$. The presence of both hard and soft donor atoms in the backbones of unsymmetrical Schiff bases ligands, they readily coordinate with a wide range of transition metal ions [1-8]. Most of these unsymmetrical Schiff bases ligands obtained by the condensation of different types of primary amines with various ketones and aldehydes $[9,10]$ by metal-templated [11-13] or by direct syntheses $[14,15]$.

The synthesis of transition metal complexes of unsymmetrical Schiff bases ligands with nitrogen, oxygen and sulfur atoms is an important area of study with implications in bioinorganic chemistry [4,5,16], catalysis [17] and medical chemistry [18].

In this study, we report the preparation of 2-(2-amino ethylthio)aniline and 3-(2-aminopropylthio)aniline in new procedure (Scheme 1) and four new macroacyclic pentadentate $\left(\mathrm{N}_{2} \mathrm{O}_{2} \mathrm{~S}\right)$ unsymmetrical Schiff base ligands by direct condensation of the 2-(2-aminoethylthio)aniline or 3-(2aminopropylthio)aniline and 2-hydroxybenzaldehyde or 5bromo-2-hydroxybenzaldehyde (Scheme 2). Zn(II) and Cd(II) complexes of these unsymmetrical Schiff base ligands have been synthesized.

\section{Experimental}

\subsection{Chemical and starting materials}

2-Aminothiophenol, 2-hydroxybenzaldehyde, 5-bromo-2hydroxybenzaldehyde, $\mathrm{Zn}\left(\mathrm{NO}_{3}\right)_{2} \cdot 6 \mathrm{H}_{2} \mathrm{O}$ and $\mathrm{Cd}\left(\mathrm{NO}_{3}\right)_{2} \cdot 4 \mathrm{H}_{2} \mathrm{O}$ were purchased from Merck and used as received. Other reagents and solvents used were of analytical grade and purchased commercially.

\subsection{Instrumentation}

Elemental analyses were performed in a CHNS-O-2400 II Perkin-Elmer. Infrared spectra were recorded in ATR, using a Bruker FT-IR Equinax-55 spectrophotometer (4000-400 $\left.\mathrm{cm}^{-1}\right)$. Mass spectra were obtained using a QP-1100EX Shimadzu GCMS (EI at $70 \mathrm{eV}$ ). ${ }^{1} \mathrm{H}$ and ${ }^{13} \mathrm{C}$ NMR spectra were taken in $\mathrm{CDCl}_{3}$ on a Bruker NMR $500 \mathrm{MHz}$ spectrometer using $\mathrm{Si}\left(\mathrm{CH}_{3}\right)_{4}$ as an internal standard. 


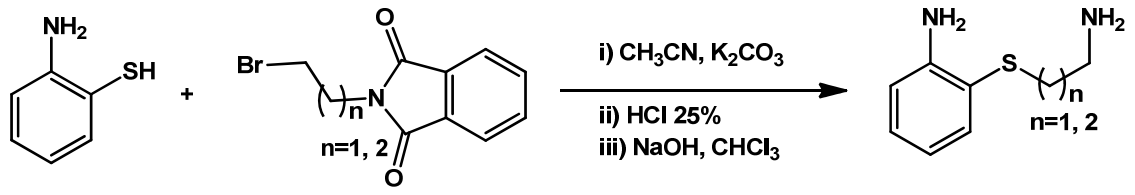

Scheme 1

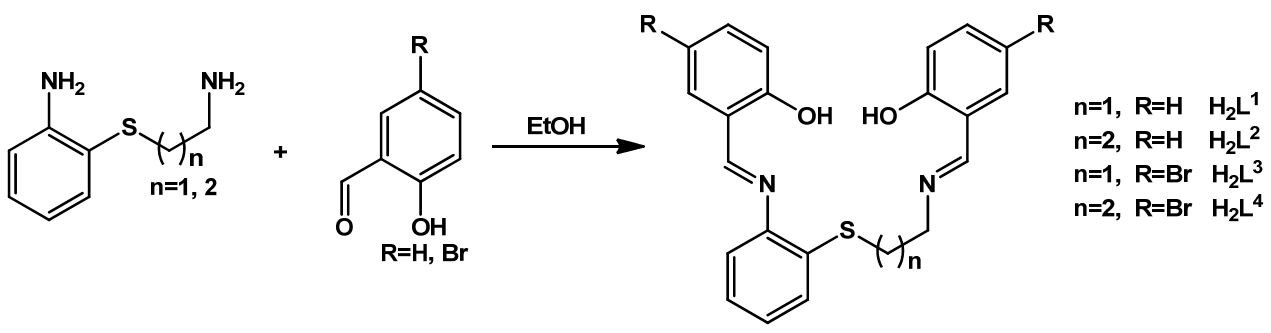

Scheme 2

\subsection{General synthesis of the thioether diamines}

2-Aminothiophenol (6.25 g, $50 \mathrm{mmol})$ was dissolved in acetonitrile $(40 \mathrm{~mL})$ and $\mathrm{K}_{2} \mathrm{CO}_{3}(6.9 \mathrm{~g}, 50 \mathrm{mmol})$ was added. The mixture was refluxed and then a solution of $N$-(2-bromo ethyl)phthalimide (12.65 g, $50 \mathrm{mmol}$ ) or $N$-(3-bromopropyl) phthalimide $(13.4 \mathrm{~g}, 50 \mathrm{mmol})$ in acetonitrile $(40 \mathrm{~mL})$ was added. The mixture was refluxed for $8 \mathrm{~h}$ and then filtered hot. The filtrate was reduced to dryness by rotary evaporation. The resulting product was boiled under reflux for $8 \mathrm{~h}$ in aqueous $\mathrm{HCl}(25 \%, 100 \mathrm{~mL})$. After this time the solution was evaporated to small volume (ca. $25 \mathrm{~mL}$ ) under vacuum and cooled in refrigerator for several hours. The solid present was filtered off, and the filtrate was evaporated to dryness under vacuum. Water $(50 \mathrm{~mL})$ was added to the mixture and the $\mathrm{pH}$ was adjusted to 13 with sodium hydroxide. The product was extracted with chloroform $(3 \times 25 \mathrm{~mL})$; the combined chloroform solutions were separated and dried over magnesium sulphate. The chloroform was removed by rotary evaporation to leave brown oil (Scheme 1).

2-(2-Aminoethylthio)aniline: Yield: 5.63 g (67\%). FT-IR (ATR, v, cm $\left.{ }^{-1}\right)$ : 3430, 3352, $3172\left(\mathrm{NH}_{2}\right), 750(\mathrm{C}-\mathrm{S}) .{ }^{1} \mathrm{H}$ NMR $(500$ $\left.\mathrm{MHz}, \mathrm{CDCl}_{3}, \delta, \mathrm{ppm}\right): 2.81\left(\mathrm{~s}, 4 \mathrm{H}, \mathrm{NCH}_{2} \mathrm{CH}_{2} \mathrm{~S}\right), 3.30-5.20(\mathrm{br}, 4 \mathrm{H}$, $\left.\mathrm{NH}_{2}\right), 6.68(\mathrm{t}, 1 \mathrm{H}, J=7.3 \mathrm{~Hz}, \mathrm{Ar}-H), 6.72(\mathrm{~d}, 1 \mathrm{H}, J=8.0 \mathrm{~Hz}, \mathrm{Ar}-H)$, $7.12(\mathrm{t}, 1 \mathrm{H}, J=6.6 \mathrm{~Hz}, \mathrm{Ar}-H), 7.38(\mathrm{~d}, 1 \mathrm{H}, J=6.3 \mathrm{~Hz}, \mathrm{Ar}-H) .{ }^{13} \mathrm{C}$ NMR (125 MHz, $\left.\mathrm{CDCl}_{3}, \delta, \mathrm{ppm}\right): 38.8\left(1 \mathrm{C}, \mathrm{CCH}_{2} \mathrm{~S}\right), 41.4(1 \mathrm{C}$, $\left.\mathrm{NCH}_{2} \mathrm{C}\right), 115.4,117.3,118.9,130.3,136.6,148.9$ (6C, Ar-C).

2-(3-Aminopropylthio)aniline: Yield: 6.55 g (72\%). FT-IR (ATR, v, cm-1): 3432, 3338, $3172\left(\mathrm{NH}_{2}\right), 746(\mathrm{C}-\mathrm{S}) .{ }^{1} \mathrm{H}$ NMR (500 $\left.\mathrm{MHz}, \mathrm{CDCl}_{3}, \delta, \mathrm{ppm}\right): 1.73\left(\mathrm{~m}, 2 \mathrm{H}, J=7.2 \mathrm{~Hz}, \mathrm{CCH}_{2} \mathrm{C}\right), 2.81(\mathrm{t}, 2 \mathrm{H}$, $\left.J=7.3 \mathrm{~Hz}, \mathrm{NCH}_{2} \mathrm{C}\right), 2.84\left(\mathrm{t}, 2 \mathrm{H}, J=5.8 \mathrm{~Hz}, \mathrm{CCH}_{2} \mathrm{~S}\right), 3.50-5.00(\mathrm{br}$, $\left.4 \mathrm{H}, \mathrm{NH}_{2}\right), 6.70(\mathrm{t}, 1 \mathrm{H}, J=7.5 \mathrm{~Hz}, \mathrm{Ar}-H), 6.73(\mathrm{~d}, 1 \mathrm{H}, J=8.0 \mathrm{~Hz}$, $\mathrm{Ar}-H), 7.13(\mathrm{t}, 1 \mathrm{H}, J=7.9 \mathrm{~Hz}, \mathrm{Ar}-H), 7.38(\mathrm{~d}, 1 \mathrm{H}, J=6.3 \mathrm{~Hz}, \mathrm{Ar}-H)$. ${ }^{13} \mathrm{C}$ NMR $\left(125 \mathrm{MHz}, \mathrm{CDCl}_{3}, \delta, \mathrm{ppm}\right): 32.5\left(1 \mathrm{C}, \mathrm{CCH}_{2} \mathrm{C}\right), 32.9(1 \mathrm{C}$, $\left.\mathrm{CCH}_{2} \mathrm{~S}\right), 40.9$ (1C, $\left.\mathrm{NCH}_{2} \mathrm{C}\right), 115.4,118.1,118.9,130.1,136.2$, 148.7 (6C, Ar- $C$ ).

\subsection{General synthesis of the Schiff base ligands}

A solution of 2-(2-aminoethylthio)aniline or 2-(3-amino propylthio)aniline $(5 \mathrm{mmol})$ in ethanol $(50 \mathrm{~mL})$ was added dropwise to a refluxing solution of 2-hydroxybenzaldehyde or 5-bromo-2-hydroxybenzaldehyde $(10 \mathrm{mmol})$ in the same solvent $\left(40 \mathrm{~mL}\right.$ ) and four new Schiff base ligands, $\mathbf{H}_{2} \mathbf{L}^{\mathbf{1}}-\mathbf{H}_{2} \mathbf{L}^{\mathbf{4}}$ were obtained, respectively. After refluxing for $3 \mathrm{~h}$ the solution was vacuum evaporated to yield the crude product as yellow oil. A small volume of petroleum ether was added to the residue remaining in the flask and rubbed. Then liquid was decanted and residue was evaporated until a yellow oil remained (Scheme 2).

2-((2-(2-(2-Hydroxybenzylidenamino)ethylthio)phenylimino) methyl)phenol $\left(\mathbf{H}_{2} \mathbf{L}^{1}\right)$ : Yield: $1.56 \mathrm{~g}(83 \%)$. FT-IR (ATR, $\left.v, \mathrm{~cm}^{-1}\right)$ : 2500-3000 (OH) (br), 1629, $1612(\mathrm{C}=\mathrm{N}), 750(\mathrm{C}-\mathrm{S}) .{ }^{1} \mathrm{H}$ NMR (500 MHz, $\left.\mathrm{CDCl}_{3}, \delta, \mathrm{ppm}\right): 3.27\left(\mathrm{t}, 2 \mathrm{H}, \mathrm{NCH}_{2}\right), 3.86\left(\mathrm{t}, 2 \mathrm{H}, \mathrm{CH}_{2} \mathrm{~S}\right)$, 6.8-7.5 (m, 12H, Ar-H), $8.33(\mathrm{~s}, 1 \mathrm{H}, \mathrm{CH}=\mathrm{N}), 8.60(\mathrm{~s}, 1 \mathrm{H}, \mathrm{CH}=\mathrm{N})$, 13.09 (br, $1 \mathrm{H}, \mathrm{OH}), 13.28$ (s, 1H, OH). ${ }^{13} \mathrm{C}$ NMR $\left(125 \mathrm{MHz}, \mathrm{CDCl}_{3}\right.$, $\delta$, ppm): $34.0\left(1 \mathrm{C}, \mathrm{CH}_{2} \mathrm{~S}\right), 58.7$ (1C, $\left.\mathrm{NCH}_{2}\right) ; 117.1,117.4,117.9$, $118.6,118.7,119.1,119.3,126.4,127.6,127.8,131.5,132.3$, 132.5, 132.8, 133.3, 146.6, 160.2, 160.9, (18C, Ar-C), 162.8, $165.1(2 \mathrm{C}, \mathrm{CH}=\mathrm{N})$. EI-MS $(\mathrm{m} / \mathrm{z}, \%): 376\left[\mathrm{M}^{+}, 100\right]$.

2-((2-(3-(2-Hydroxybenzylidenamino)propylthio)phenyl imino)methyl)phenol $\left(\mathbf{H}_{2} \mathbf{L}^{2}\right)$ : Yield: $1.46 \mathrm{~g}(75 \%)$. FT-IR (ATR, $v$, $\left.\mathrm{cm}^{-1}\right)$ : 2500-3000 (OH) (br), 1629, $1611(\mathrm{C}=\mathrm{N}), 750(\mathrm{C}-\mathrm{S}) .{ }^{1} \mathrm{H}$ NMR $\left(500 \mathrm{MHz}, \mathrm{CDCl}_{3}, \delta, \mathrm{ppm}\right): 2.11\left(\mathrm{~m}, 2 \mathrm{H}, \mathrm{CCH}_{2} \mathrm{C}\right), 3.03(\mathrm{t}$, $\left.2 \mathrm{H}, \mathrm{NCH}_{2}\right), 3.75\left(\mathrm{t}, 2 \mathrm{H}, \mathrm{CH}_{2} \mathrm{~S}\right), 6.80-7.40(\mathrm{~m}, 12 \mathrm{H}, \mathrm{Ar}-\mathrm{H}), 8.36(\mathrm{~s}$, $1 \mathrm{H}, \mathrm{CH}=\mathrm{N}), 8.62(\mathrm{~s}, 1 \mathrm{H}, \mathrm{CH}=\mathrm{N}), 13.29(\mathrm{br}, 1 \mathrm{H}, \mathrm{OH}), 13.39(\mathrm{~s}, 1 \mathrm{H}$, OH). ${ }^{13} \mathrm{C}$ NMR (125 MHz, $\left.\mathrm{CDCl}_{3}, \delta, \mathrm{ppm}\right): 29.7$ (1C, $\left.\mathrm{CCH}_{2} \mathrm{C}\right), 29.8$ (1C, $\left.\mathrm{CH}_{2} \mathrm{~S}\right), 57.9\left(1 \mathrm{C}, \mathrm{NCH}_{2}\right), 117.0,117.4,117.8,118.6,118.8$ $119.2,119.3,126.5,127.5,127.9,131.4,132.3,132.4,132.5$, $133.4,146.6,161.2,162.2(18 \mathrm{C}, \mathrm{Ar}-\mathrm{C}), 163.0,165.8(2 \mathrm{C}, \mathrm{CH}=\mathrm{N})$. EI-MS (m/z, \%): $390\left[\mathrm{M}^{+}, 100\right]$.

4-Bromo-2-((2-(2-(5-bromo-2-hydroxybenzylidenamino) ethylthio)phenylimino)methyl)phenol $\left(\mathbf{H}_{2} \mathbf{L}^{3}\right)$ : Yield: $2.23 \mathrm{~g}$ (86\%). FT-IR (ATR, v, cm-1): 2500-3000 (OH) (br), 1634, 1614 $(\mathrm{C}=\mathrm{N}), 757$ (C-S). ${ }^{1} \mathrm{H}$ NMR $\left(500 \mathrm{MHz}, \mathrm{CDCl}_{3}, \delta, \mathrm{ppm}\right): 3.12(\mathrm{t}, 2 \mathrm{H}$, $\left.\mathrm{NCH}_{2}\right), 3.86\left(\mathrm{t}, 2 \mathrm{H}, \mathrm{CH}_{2} \mathrm{~S}\right), 6.60-7.80(\mathrm{~m}, 10 \mathrm{H}, \mathrm{Ar}-\mathrm{H}), 8.30(\mathrm{~s}, 1 \mathrm{H}$, $\mathrm{CH}=\mathrm{N}), 8.63(\mathrm{~s}, 1 \mathrm{H}, \mathrm{CH}=\mathrm{N}), 13.21(\mathrm{br}, 1 \mathrm{H}, \mathrm{O}-\mathrm{H}), 13.32$ (s, 1H, OH). ${ }^{13} \mathrm{C}$ NMR (125 MHz, $\mathrm{CDCl}_{3}, \delta$, ppm): $33.9\left(1 \mathrm{C}, \mathrm{CH}_{2} \mathrm{~S}\right), 58.5$ (1C, $\left.\mathrm{NCH}_{2}\right), 110.1,110.7,117.7,119.1,119.3,120.1,120.6$, $126.6,127.9,128.2,132.7,133.6,134.3,134.7,135.8,146.3$, 160.1, 160.3 (18C, Ar- $C), 160.6,164.7(2 \mathrm{C}, \mathrm{CH}=\mathrm{N})$. EI-MS $(\mathrm{m} / \mathrm{z}$, $\%): 534\left[\mathrm{M}^{+}, 100\right]$.

4-Bromo-2-((2-(3-(5-bromo-2-hydroxybenzylidenamino) propylthio)phenylimino)methyl)phenol $\left(\mathbf{H}_{2} \mathbf{L}^{4}\right)$ : Yield: $1.97 \mathrm{~g}$ (72\%). FT-IR (ATR, $v, \mathrm{~cm}^{-1}$ ): 2500-3000 (OH) (br), 1632, 1612 $(\mathrm{C}=\mathrm{N}), 752(\mathrm{C}-\mathrm{S}) .{ }^{1} \mathrm{H}$ NMR $\left(500 \mathrm{MHz}, \mathrm{CDCl}_{3}, \delta, \mathrm{ppm}\right): 2.09(\mathrm{~m}$, $\left.2 \mathrm{H}, \mathrm{CCH}_{2} \mathrm{C}\right), 3.00\left(\mathrm{t}, 2 \mathrm{H}, \mathrm{NCH}_{2}\right), 3.74\left(\mathrm{t}, 2 \mathrm{H}, \mathrm{CH}_{2} \mathrm{~S}\right), 6.8-7.7(\mathrm{~m}$, $10 \mathrm{H}, \mathrm{Ar}-\mathrm{H}), 8.26(\mathrm{~s}, 1 \mathrm{H}, \mathrm{CH}=\mathrm{N}), 8,53(\mathrm{~s}, 1 \mathrm{H}, \mathrm{CH}=\mathrm{N}), 13.31(\mathrm{br}$, $1 \mathrm{H}, \mathrm{OH}), 13.39(\mathrm{~s}, 1 \mathrm{H}, \mathrm{OH}) .{ }^{13} \mathrm{C}$ NMR $\left(500 \mathrm{MHz}, \mathrm{CDCl}_{3}, \delta, \mathrm{ppm}\right):$ $29.4\left(1 \mathrm{C}, \mathrm{CCH}_{2} \mathrm{C}\right), 29.9\left(1 \mathrm{C}, \mathrm{CH}_{2} \mathrm{~S}\right), 57.8\left(1 \mathrm{C}, \mathrm{NCH}_{2}\right), 110.0,110.6$, $117.8,119.0,119.4,120.0,120.7,126.6,128.0,128.2,132.6$, $133.5,134.3,134.9,135.9,146.1,160.1,160.2$, (18C, Ar-C), $160.4,164.6(2 \mathrm{C}, \mathrm{CH}=\mathrm{N})$. EI-MS $(\mathrm{m} / \mathrm{z}, \%): 548\left[\mathrm{M}^{+}, 100\right]$. 


\subsection{General synthesis of the complexes}

A solution of $\mathrm{Zn}\left(\mathrm{NO}_{3}\right)_{2} \cdot 6 \mathrm{H}_{2} \mathrm{O}$ or $\mathrm{Cd}\left(\mathrm{NO}_{3}\right)_{2} \cdot 4 \mathrm{H}_{2} \mathrm{O}(1 \mathrm{mmol})$ and $\mathrm{NEt}_{3}(2 \mathrm{mmol})$ in ethanol $(30 \mathrm{~mL})$ was added to a refluxing solution of $\mathrm{H}_{2} \mathrm{~L}^{\mathrm{n}}(\mathrm{n}=1,2,3,4)(1 \mathrm{mmol})$ in the same solvent $(30$ $\mathrm{mL}$ ) and the reaction mixture was refluxed for $2 \mathrm{~h}$. The reaction mixture was then concentrated to $c a .5-10 \mathrm{~mL}$. The complexes were filtered off, washed with cold ethanol and air-dried.

[Zn( $\left.\left.\mathrm{HL}^{1}\right)\right] \mathrm{NO}_{3} \cdot \mathrm{EtOH}$ : Yield: $252 \mathrm{mg}(46 \%)$. FT-IR (ATR, $v$, $\left.\mathrm{cm}^{-1}\right): 1607$ (C=N), $754(\mathrm{C}-\mathrm{S}) .{ }^{1} \mathrm{H}$ NMR $\left(500 \mathrm{MHz}^{\mathrm{C}} \mathrm{CDCl}_{3}, \delta\right.$, ppm): $3.23\left(\mathrm{t}, 2 \mathrm{H}, \mathrm{NCH}_{2}\right), 3.83\left(\mathrm{t}, 2 \mathrm{H}, \mathrm{CH}_{2} \mathrm{~S}\right), 6.40-7.70(\mathrm{~m}, 12 \mathrm{H}$, $\mathrm{Ar}-\mathrm{H}$ ), $8.10(\mathrm{br}, 2 \mathrm{H}, \mathrm{CH}=\mathrm{N}), 10.90(\mathrm{br}, 1 \mathrm{H}, \mathrm{OH})$. EI-MS $(\mathrm{m} / \mathrm{z}, \%)$ : $439\left[\mathrm{Zn}\left(\mathrm{HL}^{1}\right)\right]^{+}$. Anal. calcd. for $\mathrm{C}_{24} \mathrm{H}_{25} \mathrm{~N}_{3} \mathrm{O}_{6} \mathrm{SZn}: \mathrm{C}, 52.51 ; \mathrm{H}$, 4.59; N, 7.65. Found: C, 51.78; H, 4.30; N, 7.77\%.

[Zn( $\left.\left.\mathrm{HL}^{2}\right)\right] \mathrm{NO}_{3} \cdot \mathrm{EtOH}$ : Yield: $293 \mathrm{mg}(52 \%)$. FT-IR (ATR, v, $\left.\mathrm{cm}^{-1}\right)$ :) $1634(\mathrm{C}=\mathrm{N}), 746(\mathrm{C}-\mathrm{S})$. EI-MS $(\mathrm{m} / \mathrm{z}, \%): 454\left[\mathrm{Zn}_{\left.\left(\mathrm{HL}^{2}\right)\right]^{+} .}\right.$ Anal. calcd. for $\mathrm{C}_{25} \mathrm{H}_{27} \mathrm{~N}_{3} \mathrm{O}_{6} \mathrm{SZn}$ : C, 53.34; H, 4.83; N, 7.46. Found: C, 53.57; H, 5.13; N, 7.69\%.

[Zn( $\left.\left.\mathrm{HL}^{3}\right)\right] \mathrm{NO}_{3} \cdot \mathrm{EtOH}$ : Yield: $465 \mathrm{mg}(66 \%)$. FT-IR (ATR, v, $\left.\mathrm{cm}^{-1}\right): 1605 \quad v(\mathrm{C}=\mathrm{N}), 754 \quad v(\mathrm{C}-\mathrm{S})$. EI-MS $(\mathrm{m} / \mathrm{z}, \%): 598$ $\left[\mathrm{Zn}\left(\mathrm{HL}^{3}\right)\right]^{+}$. Anal. calcd. for $\mathrm{C}_{24} \mathrm{H}_{23} \mathrm{Br}_{2} \mathrm{~N}_{3} \mathrm{O}_{6} \mathrm{SZn}: \mathrm{C}, 40.79 ; \mathrm{H}, 3.28$; N, 5.95\%. Found: C, 41.24; H, 3.57; N, 5.78\%.

[Zn( $\left.\left.\mathrm{HL}^{4}\right)\right] \mathrm{NO}_{3} \cdot$ EtOH: Yield: $497 \mathrm{mg}(69 \%)$. FT-IR (ATR, v, $\mathrm{cm}^{-1}$ ): 1634, 1611 (C=N), 746 (C-S). EI-MS ( $\left.\mathrm{m} / \mathrm{z}, \%\right): 611$ $\left[\mathrm{Zn}\left(\mathrm{HL}^{4}\right)\right]^{+}$. Anal. calcd. for $\mathrm{C}_{25} \mathrm{H}_{25} \mathrm{Br}_{2} \mathrm{~N}_{3} \mathrm{O}_{6} \mathrm{SZn}: \mathrm{C}, 41.66 ; \mathrm{H}, 3.50$; N, 5.83. Found: C, 41.41; H, 3.44; N, 6.04\%.

[Cd(HL')] $\mathrm{NO}_{3}$ : Yield: $435 \mathrm{mg}(79 \%)$. FT-IR (ATR, $\mathrm{v}, \mathrm{cm}^{-1}$ ):

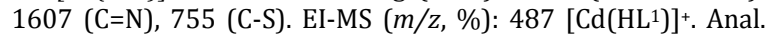
calcd. for $\mathrm{C}_{22} \mathrm{H}_{19} \mathrm{~N}_{3} \mathrm{O}_{5} \mathrm{SCd}$ : C, 48.05; $\mathrm{H}, 3.48 ; \mathrm{N}, 7.64$. Found: $\mathrm{C}$, 47.59; H, 3.42; N, 7.29\%.

[Cd(HL $\left.\left(\mathrm{HL}^{2}\right)\right] \mathrm{NO}_{3}$ : Yield: $407 \mathrm{mg}(72 \%)$. FT-IR (ATR, $\left.v, \mathrm{~cm}^{-1}\right)$ : $1622(\mathrm{C}=\mathrm{N}), 752(\mathrm{C}-\mathrm{S}) .{ }^{1} \mathrm{H}$ NMR $\left(500 \mathrm{MHz}, \mathrm{CDCl}_{3}, \delta, \mathrm{ppm}\right): 2.10$ $\left(\mathrm{m}, 2 \mathrm{H}, \mathrm{CCH}_{2} \mathrm{C}\right), 3.01\left(\mathrm{t}, 2 \mathrm{H}, \mathrm{NCH}_{2}\right), 3.79\left(\mathrm{t}, 2 \mathrm{H}, \mathrm{CH}_{2} \mathrm{~S}\right), 6.40-7.70$ $(\mathrm{m}, 12 \mathrm{H}, \mathrm{Ar}-\mathrm{H}), 8.10(\mathrm{br}, \mathrm{H}, \mathrm{CH}=\mathrm{N}), 8.30(\mathrm{br}, 1 \mathrm{H}, \mathrm{CH}=\mathrm{N}), 11.70$

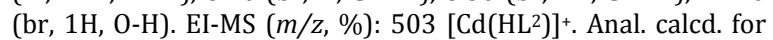
$\mathrm{C}_{23} \mathrm{H}_{21} \mathrm{~N}_{3} \mathrm{O}_{5} \mathrm{SCd}: \mathrm{C}, 48.99 ; \mathrm{H}, 3.75 ; \mathrm{N}, 7.45$. Found: C, 49.45; H, $3.72 ; \mathrm{N}, 6.93 \%$.

[Cd $\left.\left(\mathrm{HL}^{3}\right)\right] \mathrm{NO}_{3}$ : Yield: $601 \mathrm{mg}(85 \%)$. FT-IR (ATR, $\left.v, \mathrm{~cm}^{-1}\right)$ : 1610, $1620(\mathrm{C}=\mathrm{N}), 759$ (C-S). EI-MS $(\mathrm{m} / \mathrm{z}, \%): 644\left[\mathrm{Cd}\left(\mathrm{HL}^{3}\right)\right]^{+}$. Anal. calcd. for $\mathrm{C}_{22} \mathrm{H}_{17} \mathrm{Br}_{2} \mathrm{~N}_{3} \mathrm{O}_{5} \mathrm{SCd}$ : C, 37.34; $\mathrm{H}, 2.42$; N, 5.94 . Found: C, 36.90; H, 2.19; N, 6.44\%.

[Cd(HL $\left.\left.\mathrm{HL}^{4}\right)\right] \mathrm{NO}_{3}$ : Yield: $560 \mathrm{mg}(86 \%)$. FT-IR (ATR, v, $\mathrm{cm}^{-1}$ ): 1627, $1610(\mathrm{C}=\mathrm{N}), 747$ (C-S). EI-MS $(\mathrm{m} / \mathrm{z}, \%): 660\left[\mathrm{Cd}\left(\mathrm{HL}^{4}\right)\right]^{+}$. Anal. calcd. for $\mathrm{C}_{23} \mathrm{H}_{19} \mathrm{Br}_{2} \mathrm{~N}_{3} \mathrm{O}_{5} \mathrm{SCd}$ : C, 38.28; $\mathrm{H}, 2.65$; N, 5.82. Found: C, 37.99; H, 2.69; N, 5.26\%.

\section{Results and discussion}

The IR spectrum for the 2-(2-aminoethylthio)aniline shows bands at $3430,3352,3172 \mathrm{~cm}^{-1}$ and for the 2-(3-aminopropyl thio)aniline shows bands at $3432,3338,3172 \mathrm{~cm}^{-1}$ assignable to the aromatic and aliphatic primary amine stretch. The three branches peaks in the area of $3150-3450 \mathrm{~cm}^{-1}$ (due to the interaction of the two groups together, three peaks is observed) show two different groups of $\mathrm{NH}_{2}$ in the compounds. In addition, the vibration peak of C-S is observed in 750 and $746 \mathrm{~cm}^{-1}$, respectively. The IR spectra for the ligands $\left(\mathrm{H}_{2} \mathrm{~L}^{\mathrm{n}}, \mathrm{n}=\right.$ $1,2,3,4)$ confirm the presence of imines (ca. 1611-1634 $\mathrm{cm}^{-1}$ ) and the absence of carbonyl and amine functional groups of the starting materials. The total absence of $v(\mathrm{C}=0)$ absorption in the IR spectra of the ligands together with the appearance of new $v(\mathrm{C}=\mathrm{N})$ absorption in the range of $1611-1634 \mathrm{~cm}^{-1}$ clearly indicated that a new Schiff base ligand had formed in each case. In addition, the vibration peaks of C-S are observed in 750-757 $\mathrm{cm}^{-1}$. The broad band observed in the spectra of ligands in the region of $2500-3000 \mathrm{~cm}^{-1}$ can be assigned to the $v(0-\mathrm{H})$ groups. The IR spectra of $\mathrm{Zn}(\mathrm{II})$ and Cd(II) complexes of these unsymmetrical Schiff base ligands show one or two strong band at $1605-1634 \mathrm{~cm}^{-1}$ assigned to the $\mathrm{C}=\mathrm{N}$ stretching mode. These bands are shifted relative to the similar bands of the ligands that show the imines nitrogen atoms coordinated to the metal ions. The vibration peaks of C-S in complexes are observed in $746-759 \mathrm{~cm}^{-1}$.

${ }^{1} \mathrm{H}$ NMR and ${ }^{13} \mathrm{C}\{1 \mathrm{H}\}$ NMR results, obtained for some prepared compounds at ambient temperature in $\mathrm{CDCl}_{3}$, are presented in experimental section. The aliphatic protons appear as a singlet at $2.81 \mathrm{ppm}$ in 2-(2-aminoethylthio)aniline and in related ligands $\left(\mathbf{H}_{2} \mathbf{L}^{1}\right.$ and $\left.\mathbf{H}_{2} \mathbf{L}^{3}\right)$ appear as two triplet resonance in the region $\delta 3.12-3.86 \mathrm{ppm}$ but for 2-(3aminopropylthio)aniline and related ligands $\left(\mathbf{H}_{2} \mathbf{L}^{2}\right.$ and $\left.\mathbf{H}_{2} \mathbf{L}^{4}\right)$ appear as three signals in the region $\delta 1.73-3.75 \mathrm{ppm}$. The broad signal at $\delta 3.3-5.2 \mathrm{ppm}$ in the ${ }^{1} \mathrm{H}$ NMR spectra of diamines are assigned to the $\mathrm{NH}_{2}$ protons and at $\delta 13.09-13.39$ ppm in the ${ }^{1} \mathrm{H}$ NMR spectra of ligands are assigned to the $\mathrm{OH}$ protons. Signals for aromatic protons observed at $\delta$ 6.6-7.8 ppm. The ${ }^{1} \mathrm{H}$ NMR spectra of ligands, show peaks at $\delta 8.26-8.63$ ppm corresponding to the imine protons and these indicates that the condensation has occurred. The ${ }^{1} \mathrm{H}$ NMR spectra of $\mathrm{Zn}(\mathrm{II})$ and $\mathrm{Cd}(\mathrm{II})$ complexes showed that the imine signals shifted in compared to initial compound. Unfortunately, the poor solubility of the complexes made impossible there's study by NMR spectroscopy.

The EI mass spectra of the unsymmetrical Schiff bases ligands, provide strong evidence for the formation of these ligands. The peaks in the spectra of the ligands are observed at $m / z 376,390,534,548$ corresponding to $\left[\mathrm{H}_{2} \mathrm{~L}^{\mathrm{n}}\right]^{+}(\mathrm{n}=1,2,3,4)$, respectively. The spectra for the complexes exhibit peaks at higher molecular weights, confirming the presence of metal unit in the complexes.

\section{Conclusion}

We report the successful synthesis of two thioether diamines 2-(2-aminoethylthio)aniline and 2-(3-aminopropyl thio)aniline and also four potentially pentadentate $\left(\mathrm{N}_{2} \mathrm{O}_{2} \mathrm{~S}\right)$ unsymmetrical Schiff bases ligands $\left(\mathrm{H}_{2} \mathrm{~L}^{\mathrm{n}}, \mathrm{n}=1,2,3,4\right)$ have been prepared by reaction between of these diamines and 2hydroxybenzaldehyde or 5-bromo-2-hydroxybenzaldehyde. The complexation capacity of four ligands towards $\mathrm{Zn}$ (II) and Cd(II) ions has been also investigated. The complexes are mononuclear and the spectroscopic data confirm the formation of them.

\section{Acknowledgements}

We are grateful to the Faculty of Chemistry of Yazd University and Ministry of Science, Research and Technology of Iran, for financial support.

\section{References}

[1]. Lindoy, L. F. Coord. Chem. Rev. 1969, 4, 41-71

[2]. Sudbrake, C.; Vahrenkamp, H. Eur. J. Inorg. Chem. 2001, 751-754.

[3]. Masdeu-Bulto, A. M.; Dieguez, M.; Martin, E.; Gomez, M. Coord. Chem. Rev. 2003, 242, 159-201.

[4]. Taki, M.; Hattori, H.; Osako, T.; Nagatomo, S.; Shiro, M.; Kitagawa, T.; Itoh, S. Inorg. Chim. Acta 2004, 357, 3369-3381.

[5]. Dolega, A. Coord. Chem. Rev. 2010, 254, 916-937.

[6]. Canovese, L.; Chessa, G.; Visentin, F.; Uguagliati, P. Coord. Chem. Rev. 2004, 248, 945-954.

[7]. Asadi, M.; Mohammadi, K.; Esmaiezadeh, S.; Etemadi, B.; Fun, H. K. Inorg. Chim. Acta 2009, 362, 4913-4920.

[8]. Chattopadhyay, S.; Ray, M. S.; Chaudhuri, S.; Mukhopadhyay, G.; Bocelli, G.; Cantoni, A.; Ghosh, A. Inorg. Chim. Acta 2006, 359, 1367 1375.

[9]. Matsushita, T.; Shono, T. Polyhedron 1986, 5, 735-743.

[10]. Rezaeivala, M.; Keypour, H.; Salehzadeh, S.; Latifi, R.; Chalabian, F.; Katouzian, F. J. Iran Chem. Soc. 2014, 11, 431-440.

[11]. Kaasjager, V. E.; Puglisi, L.; Bouwman, E.; Driessen, W. L.; Reedijk, J. Inorg. Chim. Acta 2000, 310, 183-190.

[12]. Keypour, H.; Dehghani-Firouzabadi, A. A.; Khavasi, H. R. Polyhedron 2009, 28, 1546-1550.

[13]. Keypour, H.; Dehghani-Firouzabadi, A. A.; Rezaeivala, M.; Goudarziafshar, H. J. Iran Chem. Soc. 2010, 7, 820-824.

[14]. Chiswell, B.; Crawford, J. P.; O’Reilly, E. J. Inorg. Chim. Acta 1980, 40, 223-228. 
15]. Costes, J. P. Inorg. Chim. Acta 1987, 130, 17-21.

16]. Harrop, T. C.; Mascharak, P. K. Acc. Chem. Res. 2004, 37, 253-260.

[17]. Kwong, H. L.; Yeung, H. L.; Yeung, C. T.; Lee, W. S.; Lee, C. S.; Wong, W. L. Coord. Chem. Rev. 2007, 251, 2188-2222.

[18]. Saghatforoush, L. A, ; Chalabian, F.; Aminkhani, A.; Karimnezhad, G.; Ershad, S. Eur. J. Med. Chem. 2009, 44, 4490-4495. 